\title{
Historie der Hautklinik am DRK-Krankenhaus Chemnitz-Rabenstein
}

\section{History of the Department of Dermatology, DRK-Hospital Chemnitz-Rabenstein}

Autoren

Institute
I. Feldmann-Böddeker, J. Höpner, C. Gerlach, L. Schilde

Hautklinik (Chefärztin: Dr. med. I. Feldmann-Böddeker), DRK-Krankenhaus Chemnitz-Rabenstein
Bibliografie

DOI $10.1055 / \mathrm{s}-2007-966899$

Akt Dermatol 2007; 33:

359-361 ๑ Georg Thieme

Verlag KG Stuttgart · New York ISSN 0340-2541

Korrespondenzadresse

Dr. med. Ingrid FeldmannBöddeker

Hautklinik, DRK-Krankenhaus Chemnitz-Rabenstein Unritzstraße 23

09117 Chemnitz feldmann-boeddeker.ingrid@ drk-chemnitz.de

\section{Zusammenfassung \\ $\nabla$}

Die Hautklinik am DRK-Krankenhaus ChemnitzRabenstein entstand 1985 durch einen Umzug der Hautklinik des Bergarbeiterkrankenhauses Stollberg/Erzgebirge und die zusätzliche spätere Überleitung der Hautklinik des Krankenhauses Stadtpark/Klinikum Chemnitz.

\section{Einleitung \\ $\nabla$}

Nachdem die Stadt Chemnitz mit ihren Krankenanstalten ab dem Frühjahr 1910 nicht mehr in der Lage war, kranke Patienten aus den Umlandgemeinden und dem Bezirksverband der Amtshauptmannschaft Chemnitz aufzunehmen, wurde am 11.12.1911 der Bau eines eigenen Bezirkskrankenhauses beschlossen. In Rabenstein wurde dann für 87095 Mark ein Areal von 153000 m² $^{2}$ erworben. Auf diesem wurde in einer ca. einjährigen Bauzeit für 500000 Mark das Bezirkskrankenhaus für die Stadt Chemnitz erbaut ( $\bullet$ Abb. 1). Am 15.11.1913 erfolgte die Inbetriebnahme des Bezirkskrankenhauses mit 100 Betten und 5 Abteilungen, beschrieben werden u. a. eine Abteilung für Hautkrankheiten im Dachgeschoss sowie eine Männer- und Frauenabteilung, weiterhin eine Abteilung für Lungenkranke und eine für Kranke mit Infektionskrankheiten. In den Hauptgeschossen gab es Krankensäle mit je 13 Betten sowie Ein-, Zwei- und Dreibettzimmer. Die medizinische Leitung des Bezirkskrankenhauses wurde Herrn Oberarzt Dr. med. Richard Kanold übertragen. Ihm zur Seite standen zwei Assistenzärzte, sieben Krankenschwestern und zwei Krankenwärter. Am Montag, den 22. Dezember 1913 fand die feierliche Einweihung in Anwesenheit von König Friedrich August III. von Sachsen statt. Über die Zeit bis 1950 stehen kaum Dokumente zur Verfügung. Während des zweiten Weltkrieges gehörte das Krankenhaus Rabenstein neben seinen Aufgaben bei der Betreuung der Zivilbevölkerung, zum Reservelazarett I von Chemnitz. Am 16.4.1945 wurde das Krankenhaus Rabenstein von amerikanischen Truppen besetzt, die am 1. 7.1945 von der Roten Armee abgelöst wurden. Ab dieser Zeit war das Krankenhaus bis 1948 ein sowjetisches Militärhospital.

\section{Versorgung der Geschlechtskranken in Chemnitz 1945-1948 \\ $\nabla$}

Dokumentiert ist auch, dass 1946 der Landrat die Verlegung des Ambulatoriums für Geschlechtskrankheiten aus den Kellerräumen des DRKKrankenhauses in eine Baracke anordnete. Dieses erfolgte aufgrund eines Befehls Nr. 030 der sowjetischen Militäradministration in Deutschland, der geschaffen worden war, um ab dem 1.3.1946 die Behandlung von Geschlechtskrankheiten zu sichern. Bereits 1945 war wegen der zunehmenden Zahl an Geschlechtskrankheiten die Meldepflicht eingeführt worden und die Krankenhausbehandlung für die Lues vorgeschrieben. Im Juli 1946 gab es in Chemnitz 1750 Geschlechtskranke. Über die Versorgung dieser Patienten im DRK-Krankenhaus Chemnitz-Rabenstein liegen keine Dokumente vor. Beispielhaft soll aber erwähnt werden, dass damals in Chemnitz ein Krankenhaus am Stadtpark mit 650 Betten, vorwiegend für Haut- und Geschlechtskranke, eingerichtet wurde. In diesem Krankenhaus gab es u.a. eine Station für grob asoziale Geschlechtskranke, die Tag und Nacht von einem Volkspolizisten mit Wachhund vor der Tür bewacht wurden. Die Patienten lagen in 16-Betten-Sälen in Doppelstockbetten auf Strohsäcken. Im Keller dieses Krankenhauses gab es nur einen Duschraum mit 6 Kabinen. Die Volkspolizei blockierte zu jeder Zeit regelmäßig ohne Voranmeldung die Vergnügungslokale der Stadt und verlud jeden Lokalbesucher in Omnibusse, die bereits be- 


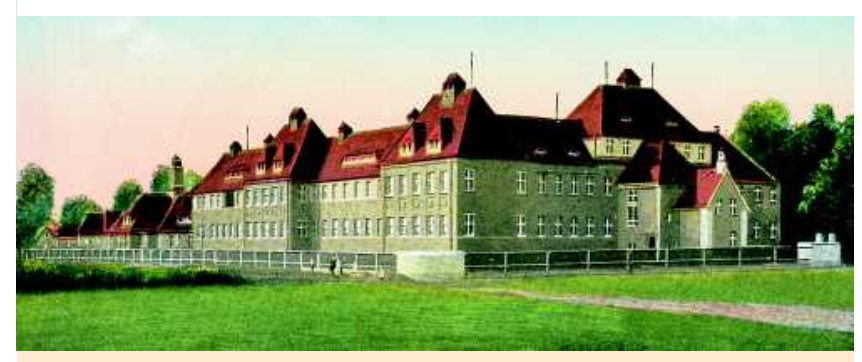

Abb. 1 Bezirkskrankenhaus der Amtshauptmannschaft ChemnitzRabenstein 1913.

reitstanden. Anschließend wurden die Lokalbesucher im Krankenhaus von der Volkspolizei in Empfang genommen und schließlich untersucht. Durch diese und auch andere Maßnahmen gelang es die Zahl der Geschlechtskranken drastisch zu reduzieren. Dadurch konnte sich aus der notdürftig eingerichteten Klinik für Haut- und Geschlechtskrankheiten im Laufe der Jahre durch Um- und Ausbauarbeiten, Modernisierung der Arbeitsbereiche und Verbesserung der Aufenthaltsbedingungen für Patienten eine anerkannte dermatologische Klinik für Chemnitz und Umgebung entwickeln. Im Jahr 1992 wurde die Hautklinik vom Krankenhaus am Stadtpark in der Scheffelstraße an das DRK-Krankenhaus Chemnitz-Rabenstein übergeleitet.

\section{Umzug der Hautklinik von Stollberg nach Chemnitz-Rabenstein \\ $\nabla$}

Im November 1948 wurde das jetzige Kreiskrankenhaus Rabenstein zum Zentralkrankenhaus der sowjetischen Aktiengesellschaft Wismut, welches von nun an nur Sowjetbürger behandelte. Auch waren jetzt überwiegend Sowjetbürger als Angestellte tätig. Im März 1957 wurde das Zentralkrankenhaus aufgelöst und ab dem 16.5.1957 vom Gesundheitswesen Wismut als Bergarbeiterkrankenhaus übernommen. Das Gesundheitswesen Wismut unterstand dem Gesundheitsministerium der DDR und sollte für die Betreuung kranker deutscher Bergarbeiter des Uranerzbergbaues im Erzgebirge zuständig sein. Jetzt wurde durch das Krankenhaus auch wieder zunehmend die Bevölkerung der umliegenden Städte und Gemeinden versorgt. Dies zeigt sich auch durch die zu diesem Zeitpunkt erfolgte Einrichtung von Kliniken für Gynäkologie und Geburtshilfe und Kinderheilkunde. 1969 verlor das Bergarbeiterkrankenhaus Rabenstein auf Beschluss des Betriebsgesundheitswesens Wismut seine Selbstständigkeit und wurde zusammen mit dem Bergarbeiterkrankenhaus Stollberg in den Medizinischen Versorgungsbereich (MVB) Karl-Marx-Stadt/Stollberg integriert. Die Folge dieses Zusammenschlusses war für das Bergarbeiterkrankenhaus Rabenstein der Verlust der Chirurgischen Abteilung bereits im Jahre 1969. Innerbetriebliche Umstrukturierungen führten 1984/1985 zur Konzentration der Inneren Medizin im Bergarbeiterkrankenhaus Stollberg und damit zur Schließung der Inneren Klinik im Bergarbeiterkrankenhaus Rabenstein. Im Gegenzug dazu wurde die Verlagerung der Hautabteilung des Bergarbeiterkrankenhauses Stollberg, welche dort seit 1949 existierte, in das Bergarbeiterkrankenhaus Rabenstein vollzogen.

\section{Die Zeit nach der Wende und Überleitung der Hautklinik Stadtpark, Scheffelstraße (Klinikum Chemnitz) \\ $\nabla$}

Mit der Wende 1989 begann für das Bergarbeiterkrankenhaus Rabenstein eine sehr bewegte Zeit. Ende 1990 wurde nach Auflösung des Gesundheitswesens Wismut das Krankenhaus über Nacht für juristisch selbstständig erklärt und war ab Januar 1990 ohne Krankenhausträger. Durch den Einsatz der damaligen Chefärzte, Herrn Dr. med. Funke und Herrn Dr. med. Viertel, gelang es, Kontakte zum Deutschen Roten Kreuz, speziell zum Verband der Schwesternschaften des DRK e.V., zu knüpfen. Im Dezember 1990 wurde die Bereitschaft zur Übernahme des Bergarbeiterkrankenhauses Rabenstein durch den Verband der Schwesternschaften des DRK e.V. erklärt.

Am 11. Januar 1991 gab die Stadt Chemnitz schriftlich ihre Zustimmung zur Übernahme des Krankenhauses in die Trägerschaft des DRK.

In diesem Zusammenhang wurde erörtert, die zum Klinikum Chemnitz (ehemals Bezirkskrankenhaus Karl-Marx-Stadt) gehörende Hautklinik der Stadt Chemnitz im Krankenhaus Stadtpark in der Scheffelstraße (Chefarzt: MR Dr. med. Hermann Müller) mit damals 109 Betten dem Krankenhaus Rabenstein zu übergeben, da ohnehin für das Gebäude Rückübertragungsansprüche seitens eines Industrieunternehmens erhoben wurden und die Einrichtung als Krankenhaus nicht mehr zur Verfügung stehen konnte.

Im Ergebnis der Beratung wurde der Standort Rabenstein als Krankenhaus der Regelversorgung mit den Fachabteilungen Gynäkologie und Geburtshilfe (60 Betten), Kinderheilkunde (45 Betten), Innere Medizin (60 Betten), Dermatologie (60 Betten) und Chirurgie (50 Betten) sowie einer Krankenpflegeschule mit je 21 Ausbildungsplätzen pro Jahrgang bestätigt und in den Krankenhausplan des Freistaates Sachsen übernommen. Im Juli 1991 konnte dann nach intensiver Vorbereitungsarbeit die DRK Gemeinnützige Krankenhaus GmbH Sachsen gegründet werden. Das nunmehrige DRK-Krankenhaus Chemnitz-Rabenstein war die erste Gesundheitseinrichtung unter dieser freien gemeinnützigen Trägerschaft in den neuen Bundesländern. Zusätzlich erfolgte auch als ein besonderes Ereignis die Gründung der Schwesternschaft des DRK Sachsen e.V.

\section{Chefärzte der Hautklinik bis 1994 \\ $\nabla$}

Über den Verbleib einer Hautabteilung seit Eröffnung des Rabensteiner Krankenhauses 1913 liegen keine Dokumente vor. In der Zeit nach dem 2. Weltkrieg hat es bis 1984 in diesem Krankenhaus keine Hautabteilung gegeben. Wie bereits erwähnt, befand sich die Hautabteilung des Betriebsgesundheitswesens Wismut von 1949 bis 1984 im Bergarbeiterkrankenhaus Stollberg. Chefärzte waren von 1949 bis 1974 Dr. Werner Sturm (1908 bis 1974), welcher vorher 11 Jahre lang als Oberarzt an der Universitätshautklinik Jena tätig gewesen war, und ab 1974 Frau MR Hannelore Rennau-Lange. Nach erfolgtem Umzug befand sich die Dermatologische Abteilung mit 42 Betten ab 1.1.1985 im Bergarbeiterkrankenhaus Rabenstein. Als Chefärztin fungierte weiterhin bis 1994 Frau MR Hannelore Rennau-Lange.

1986 wurde das Dermatologen-Ehepaar Raith von der Hautklinik der Medizinischen Akademie Erfurt in das Bergarbeiterkrankenhaus eingestellt. Daraufhin wurde als besonderer Schwerpunkt der Dermatologischen Abteilung zusätzlich ein Chefarzt- 


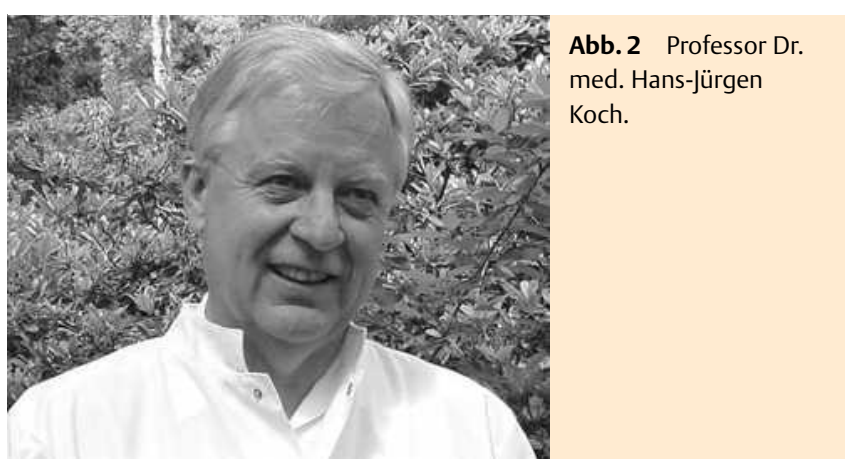

bereich Arbeitsdermatologie unter Leitung von MR Dr. Ludwig Raith geschaffen. Frau Dr. Margot Raith wurde Oberärztin und Leiterin der Hautambulanz. Das Ehepaar Raith verließ 1991 Rabenstein und ging in die Niederlassung.

Nach Übernahme des Krankenhauses in die Trägerschaft des DRK und der Aufnahme in den Krankenhausplan des Freistaates Sachsen wurde im Oktober 1992 dem Fachgebiet Dermatologie der gesamte Versorgungsauftrag für Chemnitz und die angrenzenden Landkreise zugesprochen.

\section{Sanierung und Entwicklung der Hautklinik seit 1994 \\ $\nabla$}

Am 1. 2.1994 übernahm Herr Prof. Dr. med. Hans-Jürgen Koch, 1943 in Breslau geboren, promoviert und habilitiert an der Hautklinik der Universität Jena, als Chefarzt die Leitung der Hautklinik ( Abb. 2). Herr Professor Koch war zuvor von 1985 bis 1994 als Chefarzt der Hautklinik des Bezirkskrankenhauses Plauen tätig. 1998 wurde er Privatdozent an der Universität Leipzig und 2002 zum außerplanmäßigen Professor an der Medizinischen Fakultät der Universität Leipzig berufen.

Von 1994 bis 1996 wurde das alte Hauptgebäude des DRK-Krankenhauses einer aufwendigen Sanierung und Umgestaltung unterzogen. Mit 17 Mio. Mark wurde die Hautklinik als Abteilung der Schwerpunktversorgung für den Großraum Chemnitz eingerichtet. Unter der Leitung von Professor Koch war kontinuierlich eine positive Entwicklung der Hautklinik Chemnitz-Rabenstein zu verzeichnen. Insbesondere der Aufbau einer modernen leistungsfähigen Dermatochirurgie und Dermatoonkologie als eigener Funktionsbereich ist hervorzuheben. Während seiner Zeit erfolgte der Einbau von zwei Eingriffsräumen für die operative Dermatologie. Die Einrichtung eines immunologischen und immunhistologischen Labors sowie die Erweiterung des allergologischen Labors verbesserten die diagnostischen Möglichkeiten immunologischer und allergologischer Erkrankungen. Die Fotodiagnostik, Fototherapie, Lasertherapie, Arbeitsdermatologie und das Begutachtungswesen sowie die pädiatrische Dermatologie in Kooperation mit der Kinderklinik des Hauses stellten weitere Betreuungsschwerpunkte dar.

1995 rekonstruierte man die beiden Stationen der Dermatologischen Abteilung im Erdgeschoss und im Obergeschoss mit je 30 Betten. Hierbei entstanden Ein- bis Vierbettzimmer mit eigenen Nasszellen. Vorher gab es drei Toiletten für 15 Patienten.

Von 1998 bis 2002 war Herr Professor Dr. Koch zusätzlich als Leitender Chefarzt des DRK-Krankenhauses Chemnitz-Rabenstein tätig. Stellvertretend für die zahlreichen akademischen Lehrveranstaltungen und Fortbildungsveranstaltungen, die Herr Professor Dr. Koch organisiert und geleitet hat, sollen die jährli- chen Dreiklinikstagungen der Hautkliniken Plauen, Zwickau und Chemnitz, die unter seiner Regie turnusgemäß 1998, 2001 und 2004 durchgeführt wurden, genannt werden. Herr Professor Dr. Koch richtete die 2. Gemeinschaftstagung der Sächsischen Gesellschaft Dermatologie und des Landesverbandes Sachsen im Berufsverband der Deutschen Dermatologen im November 1995 in Oberwiesenthal aus.

Über viele Jahre hat sich Herr Professor Dr. Koch als Mitglied in den Vorständen der Sächsischen Gesellschaft für Dermatologie und Venerologie und des Berufsverbandes der Dermatologen Sachsens sowie als Leiter der Fachkommission Dermatologie und Venerologie der Landesärztekammer Sachsen um die Akzeptanz und den Bestand in unserem Fachgebiet in seiner ganzen Breite große Verdienste erworben. Am 8. Oktober 2005 verstarb Herr Professor Dr. Koch plötzlich und unerwartet im Alter von 62 Jahren.

Anschließend war Herr Dr. med. Jörg Höpner, Facharzt für Dermatologie und Chirurgie bis zum 31. Juli 2006 kommissarischer Chefarzt der Hautklinik am DRK-Krankenhaus Chemnitz-Rabenstein. Unter äußerst schwierigen Bedingungen (neben dem kommissarischen Chefarzt standen kein Oberarzt und lediglich vier Assistenzärzte zur Verfügung) und mit extrem hohem Engagement gelang es ihm, die Hautklinik in ihrer nahezu gesamten fachlichen Breite aufrecht zu erhalten. Herrn Dr. Höpner ist insbesondere der weitere Ausbau der Dermatochirurgie und Dermatoonkologie zu verdanken. Als langjährig erfahrener Facharzt für Chirurgie und Dermatologie führte er u.a. die Tumeszenzlokalanästhesie, die Vakuumversiegelungstechnik und Saugkürettage bei Hyperhidrosis axillaris neu in die Hautklinik ein. Am 1. 8. 2006 übergab Herr Dr. Höpner die Leitung der Hautklinik der Chefärztin Frau Dr. med. Ingrid Feldmann-Böddeker.

\section{Abstract}

\section{History of the Department of Dermatology, DRK-Hospital Chemnitz-Rabenstein} $\nabla$

The Department of Dermatology of the DRK-Hospital ChemnitzRabenstein was founded due to the move of the Department of Dermatology of the Stollberg hospital for miners (1985) and the later following connection with the Department of Dermatology of the „Stadtpark/Klinikum“ Chemnitz (1992).

\section{Literatur}

1 Höpner J. Nachruf für Prof. Dr. med. Hans-Jürgen Koch. Ärzteblatt Sachsen 2006; 1: 41-50

2 Göring HD. Prof. Dr. Koch zum 60 Geburtstag. Hautarzt 2003; 54: $1135-1136$

3 Blun R, Mischke D, Kowalzick L. 1860 - 2005: 145 Jahre stationäre Dermatologie in Plauen: Zur Geschichte der Plauener Hautklinik. Aktuelle Dermatologie 2004; 11: 469-473

4 DRK Gemeinnützige Krankenhaus GmbH Sachsen, DRK-Krankenhaus Chemnitz-Rabenstein. Patientenratgeber. Ausgabe 1999 und 2002

5 Schilde L. 90 Jahre Krankenhaus Rabenstein Teil 1. Ortsgeschichte $2005: 8-10$

6 Schilde L. 90 Jahre Krankenhaus Rabenstein Teil 2. Ortsgeschichte $2005: 8-10$

7 Schilde L. 90 Jahre Krankenhaus Rabenstein Teil 6. Ortsgeschichte 2006: $12-13$

8 Schilde L. 90 Jahre Krankenhaus Rabenstein Teil 7. Ortsgeschichte $2006: 21-23$

9 Adler H, Weber B, Jacobs D et al. Klinikum Chemnitz: 50 Jahre Krankenhaus Stadtpark. Festschrift des Klinikum Chemnitz. 1996: 18-19 